\section{Most Surgical Infections Not Detected}

Surgical site infections (SSIs) that occur after hospital discharge cause substantial morbidity, but their epidemiology is not well understood, and methods for routine postdischarge surveillance have not been validated. Dr. Kenneth Sands and colleagues from Brigham and Women's Hospital, Harvard Medical School, and Harvard Pilgrim Healthcare recently conducted a surveillance study of SSIs occurring before and after discharge among members of a health maintenance organization (HMO) for whom detailed information was available for all postdischarge medical encounters and prescriptions through extensive automated records that are completed for every patient encounter. In addition, the researchers evaluated the performance of mailed patient and surgeon questionnaires for detecting SSIs in the same cohort and estimated the resource utilization associated with these infections.

Inpatient and outpatient surveillance was conducted on 5,572 non-obstetric surgical procedures among members of the HMO. Using a computerized search, these records were screened for coded diagnoses, testing, treatment codes, and antibiotic prescriptions that were indicative of an SSI. The full text of the records of all patients identified in the search was reviewed by a physician. In addition, questionnaires regarding the occurrence of an SSI were sent to the study patients and their surgeons.

There were 132 SSIs meeting CDC criteria, of which $84 \%$ occurred after hospital discharge, and $63 \%$ were managed outside the surgical facility. Postdischarge SSIs led to an average of 4.6 additional ambulatory encounters. Patient and surgeon questionnaires had a sensitivity of $28 \%$ and $15 \%$, respectively.

These results suggest that most SSIs occur after hospital discharge and are not detectable by conventional surveillance, and that they cause substantial resource utilization. An estimated 325,000 postoperative SSIs occur each year in the United States and generate additional medical costs in the range of $\$ 1$ to $\$ 2$ billion. Routine surveillance for SSIs is recommended by the CDC and the Surgical Infections Society as a mechanism for reducing these infections, by providing feedback to surgeons.

Given decreasing lengths of stay and increasing use of ambulatory surgery, identification of SSIs will require that hospitals, managed-care organizations, and insurers perform some form of postdischarge surveillance. However, direct wound surveillance among outpatients is extremely resource-intensive, and thus many hospitals doing postdischarge surveillance have relied on responses to questionnaires mailed to either the patients or surgeons. The authors of this study point out the considerable disadvantage of mailed questionnaires to patients and surgeons, which are less accurate (lower sensitivity and lower specificity) and more resource intensive than use of automated administrative and patient-care data, which are becoming increasingly available. Further research is needed to define better the risk factors for postdischarge SSIs that might allow for a surveillance strategy that focuses on high-risk groups.
FROM: Sands K, Vineyard G, Platt R. Surgical site infections occurring after hospital discharge. I Infect Dis 1996;173:963-970.

\section{Used Syringes Provide Estimate of Hepatitis Prevalence}

Studies to determine prevalence of hepatitis B virus (HBV) and hepatitis C virus (HCV) among injection drug users often are hampered because of the users' reluctance to be tested. Dr. R. Heimer and coinvestigators from Yale University and Abbott Laboratories recently conducted preliminary studies to evaluate the usefulness of testing used syringes for $\mathrm{HBV}$ and $\mathrm{HCV}$, using both enzyme immunoassay (EIA) and polymerase chain reaction (PCR) techniques, using the results to estimate the prevalence of $\mathrm{HBV}$ and $\mathrm{HCV}$ infection among injection drug users.

The researchers found that assays of HBV DNA and HCV RNA using PCR were orders of magnitude more sensitive than testing for antibodies to $\mathrm{HBV}$ and $\mathrm{HCV}$ using standard EIA techniques. The authors concluded that testing syringes can help estimate the prevalence and incidence of hepatitis virus infections when standard seroepidemiologic analysis cannot be applied.

FROM: Heimer R, Khoshnood K, Jariwala-Freeman B, et al. Hepatitis in used syringes: the limits of sensitivity of techniques to detect hepatitis B virus (HBV) DNA, hepatitis $\mathrm{C}$ virus (HCV) RNA, and antibodies to $\mathrm{HBV}$ core and HCV antigens. J Iinfect Dis 1996;173:997-1000.

\section{Ebola Virus Infects Monkeys in Texas}

A monkey imported from the Philippines and being held in a government animal quarantine facility in Texas died on March 30, 1996, following a 3-day illness. Ebola virus infection was confirmed as the cause of death on April 11,1996 , based on antigen detection from a liver specimen. On April 9, a second monkey that had been held in the same room had an onset of similar symptoms, was euthanized, and also was found to have Ebola infection. Sequence analysis of the gene of the Ebola virus from the first monkey indicated a $98.9 \%$ nucleotide identity with the original 1989 Ebola-Reston virus associated with an outbreak of hemorrhagic fever in a research facility in Reston, Virginia. This outbreak was made famous by Richard Preston in his best-selling book The Hot Zone.

The other 48 monkeys housed in the same quarantined room as the two infected monkeys were euthanized to minimize the potential exposure of employees and to prevent additional transmission within the room.

Ebola virus was discovered in 1976. Since its discovery, four distinct subtypes have been identified: Zaire, Sudan, Ivory Coast, and Reston. The 1989 outbreak of Ebola-Reston subtype also involved monkeys imported from the Philippines. Although infection with the EbolaReston virus subtype can be fatal in monkeys, the only four infections confirmed in humans were asymptomatic. In 
contrast, infection with Ebola-Sudan or Ebola-Zaire subtypes often is fatal in humans. Four additional episodes of Ebola Reston infection among monkeys imported from the Philippines have occurred in the United States and Italy. The reservoir and natural history of infection with this virus needs to be characterized.

FROM: Rochell A. Virus infects monkeys in Texas; strain so far harmless to humans. Atlanta Constitution April 18, 1996; A1, and Centers for Disease Control and Prevention. Ebola-Reston virus infection among quarantined nonhuman primates-Texas, 1996. MMWR 1996;45:314-316.

\section{Acceptance of INH by Healthcare Workers}

$\mathrm{CDC}$ recommends that healthcare workers ( $\mathrm{HCWs)}$ with a recent tuberculin skin-test conversion be offered preventive therapy (usually isoniazid [INH] for 6 months), which has been shown to reduce greatly the risk of developing active disease after tuberculosis infection. Despite the benefits of preventive therapy, previous studies have reported very poor physician compliance with preventive therapy. In addition, in many institutions, there have been no formal or mandatory skin-testing programs and no established protocols for referrals for those with tuberculosis infection.

Bernard Camins and colleagues recently studied the acceptance of and adherence to preventive therapy by healthcare workers (hospital employees, house staff, and medical students) at Grady Memorial Hospital in Atlanta, a university-affiliated, public, inner-city hospital with a mandatory skin-testing program that included physician referrals for all workers with positive tuberculin skin test (TST).

A total of 125 HCWs (91 hospital employees and 34 house staff or medical students) with a recent TST conversion were offered INH prophylaxis. All were required to have a chest x-ray, but were encouraged only to undergo preventive therapy. Of the $125 \mathrm{HCW}$ sith TST conversion, 105 (84\%) initiated preventive therapy. Sixty-nine (66\%) of the $105 \mathrm{HCWs}$ who initiated therapy (55\% of the 125 total) completed at least 6 months of isoniazid therapy. More of the physician group than of the employee group completed preventive therapy (25 of 34 [74\%] versus 44 of 91 [48\%], respectively). Of the $36 \mathrm{HCWs}$ who started but did not complete preventive therapy, 12 discontinued therapy because of an adverse drug effect, and 24 were noncompliant.

The authors concluded that acceptance of tuberculosis preventive therapy by HCWs was high in the setting of a comprehensive TST program, and completion of therapy was much higher in the physician group than in previous reports.

FROM: Camins BC, Bock N, Watkins DL, Blumberg HM. Acceptance of isoniazid preventive therapy by health care workers after tuberculin skin test conversion. JAMA 1996;275:1013-1015.

\section{Heterosexual Transmission Varies by HIV Strain}

Dr. Soto-Ramirez and colleagues recently reported that some strains of HIV-1 have more potential for heterosexual transmission than others. Vaginal intercourse is the most common route of transmission in Thailand, subSaharan Africa, and India, where HIV-1 subtype E is the predominant strain. Most transmissions in the United States and western Europe are associated with anal intercourse among homosexual men or with injection drug use, and HIV-1 subtype B is the most common strain. Epithelial Langerhans' cells (LCs), which express CD4 on their membranes, are present on oral and genital mucosal surfaces. They also are abundant in the cervix, but absent from the rectal mucosa, and are a possible source of contact for heterosexual transmission. The researchers found that HIV subtype E from samples taken from homosexual men in the United States grew more efficiently in Langerhans cells than HIV subtype B samples taken from heterosexuals in Thailand.

The findings suggests that Langerhans cells are primary targets for heterosexual infection, which could explain the differences in the epidemics in Asia and Africa, compared to those in the United States. Researchers in the United States expressed concern over the findings, because HIV subtype $\mathrm{E}$ has been detected in US servicemen returning from overseas duty. The fear is that if HIV subtype $\mathrm{E}$ is introduced in the West, it could represent a significantly greater threat than subtype B among heterosexuals.

FROM: Soto-Ramirez LE, Renjifo B, McLane MF, et al. HIV-1 Langerhans' cell tropism associated with heterosexual transmission of HIV. Science 1996;271:1291-1294.

\section{Globulin Protects Against RSV}

The FDA has licensed Respiratory Syncytial Virus Immune Globulin Intravenous (RSV-IGIV) to protect infants against serious effects of RSV disease. RSV-IGIV (Respigam) is manufactured by Massachusetts Public Health Biologic Laboratories in Boston and distributed by Medimmune Inc, Gaithersburg, Maryland. It is indicated for prevention of serious lower respiratory tract infections caused by RSV in children younger than 24 months with bronchopulmonary dysplasia or infants younger than 1 year with a history of premature birth. Although it does not prevent RSV infections, it has been demonstrated to be safe and effective in reducing the incidence and duration of RSV hospitalization and the severity of RSV illness in high-risk infants.

Like other plasma products, RSV-IGIV carries the possibility of transmission of bloodborne pathogens. The risk is low, however, because plasma donors are screened carefully, and a solvent detergent viral procedure inactivates most known bloodborne viruses, including HIV-1, hepatitis $B$ virus, and hepatitis $C$ virus.

FROM: Nightingale S. From the FDA: first product available for preventing serious RSV disease. JAMA 1996;275:902. 